\title{
Mix design method for self compacting metakaolin concrete with different properties of coarse aggregate
}

\author{
O.R. Khaleel, H. Abdul Razak
}

The properties of concrete depend on many factors and therefore all these factors should be taken into consideration during the process of mix design. These factors include the source and type of the constituent materials and their properties and how they can be combined. Therefore, the constituents of concrete should be selected carefully because the influence of individual constituents on self-compacting concrete (SCC) is greater than compared to normal concrete. Therefore, strict limits should be adopted with regards to the properties and mix proportions of these constituents.

Dinakar et al. [1] mentioned that the mix design method for SCC is not based on the strength requirement like normal concrete. Okamura and Ouchi [2] reported that the self compactability of concrete can be achieved by having a balance between the flowability and viscosity of paste or mortar, so that the increase of flowability is necessary to reduce the friction between aggregate particles. At the same time the presence of proper viscosity is also necessary in order to avoid any segregation between the constituents of mixes particularly as the aggregate passes through the steel bars. The flowability can be increased by using HRWR and increasing viscosity either with a viscosity modifying agent, a mineral admixture, or both. Moreover, reducing the coarse aggregate volume in the mix can reduce the probability of collision between aggregate particles and thus increase the flowability, passing ability and stability of the mix. Researchers have found that high content of coarse aggregate would consume greater energy for flowability.

When designing the mix, it is quite useful to put into consideration the relative proportions of the key constituents by volume rather than mass. Typical ranges of quantities and proportions that can help achieve self-compactability are discussed later. However, further modifications might be required to meet strength and other requirements. Water/powder ratios by volume should range between 0.80 and 1.10 , while water content does not normally exceed $200 \mathrm{l} / \mathrm{m} 3$. Total cementitious content should be within $160-240$ I, i.e. $400-600 \mathrm{~kg}$ per m3. Normally coarse aggregate content is between $28 \%$ and $35 \%$ by volume of the mix. Finally, the fine aggregate content balances the volume of the other components. This previous method is based on an improved method proposed by Okamura and Ozawa [3]. 
The coarse aggregate content in SCC is vital in ensuring that the

mix has proper mechanical properties and sufficient flow characteristics

$[4,5]$. A high coarse aggregate content can lead to a decrease

in segregation resistance leading to flow blockages $[5,6]$.

Aggregates may have a significant influence on the properties of

hardened concrete. The deformation properties of concrete are significantly influenced by aggregates due to a combination of the effects which include volume concentration, water demand, aggregate

stiffness and paste/aggregate interaction [7]. Aïtcin [8] deduced

that it is important to note that, although an excess of

coarse aggregate could decrease drying shrinkage, it will however

increase the amount of micro-cracks within the paste. Tviksta [9]

also stated that it is possible to use naturally rounded, crushed,

or semi-crushed aggregates to make SCC. Combined aggregate

grading is the most important aspect of making an SCC mix, but

a good gradation of particles helps to stop segregation [10]. A

well-graded aggregate source can be a key factor in successfully

producing an economical SCC. SCC can be produced with poorly

graded aggregate, but requires additional viscosity to prevent segregation

[11]. Hu and Wang [12] came to the conclusion that

graded aggregate can considerably reduce yield stress and viscosity

of concrete. The objective of this study is to evaluate the mix design

method to produce SCC with different properties of coarse

aggregate.

Experimental work

Materials

In this study, two types of powder materials were used namely

Type I ordinary Portland cement and metakaolin. The physical and

chemical properties of these materials are listed in Table 1. The

particle size distribution of cement and metakaolin are shown in

Fig.1. The chemical admixture used was a modified polycarboxylate

superplasticizing admixture with solid content $30 \%$ and specific

gravity of 1.05 . Silica sand was used as fine aggregate with water

absorption $0.35 \%$, a fineness modulus of 2.5 and specific gravity

of 2.64. The coarse aggregate used was crushed granite with two

different particle shapes, i.e. angular as well as a semi rounded,

and rough surface. Three types of grading and maximum sizes

were used namely continuous grade and gap graded sizes between

19 and $4.75 \mathrm{~mm}$, continuous grade and single graded sizes between

14.5 and $4.75 \mathrm{~mm}$ and single grade of sizes between 9.5

and $4.75 \mathrm{~mm}$. The sieve analysis for the types of coarse aggregates

used is shown in Table 2. The apparent specific gravity of coarse

aggregate is 2.61 and water absorption is $0.65 \%$.

Mix proportions

The mix design adopted for this study was divided into three 
phases. The first phase involved cement paste design. The second phase the design for mortar, and thirdly the design for concrete.

(a) Assume the air content (by volume of concrete).

(b) Designing of cement paste composition.

(c) Adjusting of the sand mortar ratio and paste content.

(d) Determining of the optimum replacement level of mineral admixture with cement.

(e) Determining of coarse aggregate volume.

The details of mix design method are as follows:

(a) Assume the air content (by volume of concrete).

The air content was fixed so that it does not exceed $2 \%$.

(b) Designing of cement paste.

The cement paste is thought of as the medium which transports the aggregate. Thus, achieving self compactability of the cement paste is necessary in order to achieve the self compactability of SCC. Therefore, there must be balance between the flowability and viscosity of paste by selecting the proper ratio or dosage of $w / p$ and superplasticizer respectively. The cement paste design was based on the EFNARC guidelines [13]. The paste cement design includes the determination of $w / c$ ratio and optimum dosage of superplasticizer of neat cement mixes.

The w/c ratio was determined for all mortar, while for concrete mixes, the $w / p$ ratio was ascertained. The specified $w / c$ ratio was in accordance with the paste design method of EFNARC [13] whereby the selected $w / c$ represents the minimum water demand for cement particles to allow the cement paste to initiate flow. The required $w / c$ ratio was 0.32 by weight or 0.995 by volume.

The optimum superplasticizer dosage was determined based on the results of the mini slump flow of cement paste mixes and was $1.1 \%$ of the cement weight. This dosage of superplasticizer is considered the saturation dosage for cement paste. Saturation dosage of superplasticizer itself is defined as the dosage beyond which there is no increase or change to the slump flow spread of mixes. The results of trial mixes of cement paste are shown in Section 3.1.

(c) Adjusting of the sand mortar ratio.

The optimum sand to mortar ratio was determined by trial mixes of the different sand/mortar ratio which ranged from 0.45 to 0.5 by volume of mortar. According to many studies.

\section{Full text available at :}

http://www.sciencedirect.com/science/article/pii/S0261306913007000

http://ac.els-cdn.com/S0261306913007000/1-s2.0-S0261306913007000-main.pdf? tid=1e26982e-781f-11e3860f-00000aacb360\&acdnat $=1389156259$ 76137f4da553a65b9ff2022ab26078b1 